\title{
Weld pool dynamics and the formation of ripples in 3D gas metal arc welding
}

\author{
J. Hu ${ }^{+}$, H. Guo and H.L. Tsai ${ }^{*}$ \\ Department of Mechanical and Aerospace Engineering, \\ University of Missouri-Rolla, 1870 Miner Circle, Rolla, MO 65409, United States \\ + Current address: Department of Mechanical Engineering, University of Bridgeport, \\ Bridgeport, CT 06604, United States
}

\begin{abstract}
This article studies the transient weld pool dynamics under the periodical impingement of filler droplets that carry mass, momentum, thermal energy, and species in a moving 3D gas metal arc welding. The complicated transport phenomena in the weld pool are caused by the combined effect of droplet impingement, gravity, electromagnetic force, plasma arc force, and surface tension force (Marangoni effect). The weld pool shape and the distributions of temperature, velocity, and species in the weld pool are calculated as a function of time. The phenomena of "open and close-up” for a crater in the weld pool and the corresponding weld pool dynamics are analyzed. The commonly observed ripples at the surface of a solidified weld bead are, for the first time, predicted by the present model. Detailed mechanisms leading to the formation of ripples are discussed.
\end{abstract}

Keywords: GMAW; Ripples; Weld pool dynamics

" Corresponding author: Tel: +1 573341 4945; fax: +1 573341 4607; E-mail address: tsai@umr.edu (H.L. Tsai). 


\section{Nomenclature}
$A_{v} \quad$ constant, in Eq. (21)
$\boldsymbol{B}$ magnetic flux vector
$B_{\theta}$ self-induced azimuthal magnetic field
C specific heat
$c_{1} \quad$ permeability coefficient, defined in Eq. (9)
C inertial coefficient, defined in Eq. (10)
$d$ dendrite arm spacing or electrode diameter
D mass diffusion coefficient
$D_{d} \quad$ droplet diameter

$f \quad$ mass fraction

$f^{\alpha}$ sulfur concentration

$F \quad$ volume of fluid function

$F_{d}$ droplet generation frequency

$g \quad$ volume fraction or gravitational

acceleration

$h$ enthalpy

$h_{c} \quad$ convective heat-transfer coefficient

$H \quad$ latent heat of fusion

$H_{b} \quad$ thickness of base metal

$H_{v} \quad$ latent heat of vaporization

I welding current

$\boldsymbol{J}$ current density vector

$J_{r} \quad$ r-component of $\boldsymbol{J}$

$J_{z} \quad$ z-component of $\boldsymbol{J}$

$k$ thermal conductivity

$K \quad$ permeability, defined in Eq. (9)

$L \quad$ length of base metal

$\vec{n} \quad$ normal vector to local free surface

$p \quad$ pressure

$p_{v} \quad$ vapor pressure or any other applied

external pressure
$P_{a t m}$ atmospheric pressure

$q_{\text {conv }}$ heat loss by convection

$q_{\text {evap }}$ heat loss by evaporation

$q_{\text {radi }}$ heat loss by radiation

$r \quad$ position vector from arc center

$r-Z \quad$ moving cylindrical coordinate system

$R \quad$ gas constant

$\vec{s} \quad$ tangential vector to local free surface

$S_{\phi} \quad$ source term, in Eq. (33)

$t$ time

$\delta t \quad$ time interval

$T$ temperature

$T_{l} \quad$ liquidus temperature

$T_{s} \quad$ solidus temperature

$T_{0}$ reference temperature

$T_{\infty}$ ambient temperature

$u \quad$ velocity in $x$-direction

$u_{w}$ arc voltage

$v \quad$ velocity in $y$-direction

$V_{a} \quad$ welding speed

$V_{d}$ droplet impingement velocity

$V_{w}$ wire feed rate

$\boldsymbol{V}$ velocity vector

$\tilde{\boldsymbol{V}}$ calculated temporary velocity vector, in Eq. (34)

$\boldsymbol{V}_{r}$ relative velocity vector $\left(\boldsymbol{V}_{l}-\boldsymbol{V}_{s}\right)$

$w \quad$ velocity in $z$-direction

$W$ melt mass evaporation rate or width of base metal

$x_{a} \quad$ transient arc center location in $x$-direction $x-y-z$ stationary coordinate system

Greek symbols 
$\phi \quad$ scalar electric potential

$\beta_{s} \quad$ solutal expansion coefficient

$\beta_{T} \quad$ thermal expansion coefficient

$\gamma$ surface tension

$\partial \gamma / \partial T \quad$ surface tension temperature gradient

$\partial \gamma / \partial f^{\alpha} \quad$ surface tension concentration gradient

$\varepsilon \quad$ radiation emissivity

$\kappa \quad$ free surface curvature

$\mu_{l} \quad$ dynamic viscosity of liquid metal

$\mu_{0} \quad$ magnetic permeability

$\eta \quad$ arc efficiency

$\eta_{d} \quad$ ratio of droplet thermal energy to total arc

energy

$\sigma \quad$ Stefan-Boltzmann constant

$\sigma_{e} \quad$ electrical conductivity

$\sigma_{c} \quad$ arc current distribution parameter $\sigma_{p} \quad$ arc pressure distribution parameter

$\sigma_{q}$ arc heat flux distribution parameter

$\rho$ density

$\tau \quad$ viscous stress tensor

$\tau_{\vec{s}} \quad$ Marangoni shear stress

\section{Subscripts}

$b \quad$ base metal

$d$ droplet

$l \quad$ liquid phase

$r \quad$ relative to solid phase velocity

$s \quad$ solid phase

\section{Superscripts}

$n \quad$ time step $\mathrm{n}$

$n+1$ time step $\mathrm{n}+1$

\section{Introduction}

Among the more than twenty welding methods, gas metal arc welding (GMAW) is one of the most widely used techniques in industry for joining steel, aluminum, and other alloys [1]. GMAW is a very complicated process involving many parameters, such as the power characteristics of the welding equipment, electrode type and size, shielding gas, and physical and chemical properties of the base metals. Any of these parameters can significantly affect the formation of weld beads and ultimately the weld quality. As these parameters are coupled together and since GMAW involves non-transparent metal and high temperature plasma, it is rather difficult to experimentally isolate and identify the importance of each parameter. However, it is necessary to understand the role each parameter plays on transport phenomena occurring in the weld pool in order to optimize welding conditions and maximize weld quality [2]. Mathematical modeling certainly provides a convenient means to undertake this task.

In the past, a great deal of effort was focused on the mathematical simulation of the weld pool in gas tungsten arc welding (GTAW) [e.g., 3-8]. Marangoni flow (surface tension driven flow) was found to dominate the fluid flow in the weld pool, affecting weld penetration and the formation of segregation and porosity [e.g., 9-12]. The process of GMAW is much more complicated than that of GTAW, as GMAW involves the impingement of droplets onto the weld pool, creating the mixing of mass, momentum, 
thermal energy, and species in the weld pool. Therefore, even for stationary spot GMAW, very few experimental or theoretical studies are available. Tsao and Wu [13] and Jaidi and Dutta [14] presented a two-dimensional, stationary weld pool convection model, in which the weld pool surface was assumed to be flat and mass transfer was not considered. Wang and Tsai [15] and Fan and Kovacevic [16,17] investigated the dynamic impingement of droplets onto the weld pool and the solidification process in spot GMAW. Wang and Tsai [18] then continued to investigate the Marangoni effect caused by surfaceactive elements (SAE) on weld pool mixing and weld penetration. Zhu et al. [19] and Hu and Tsai [20,21] further developed a comprehensive 2D GMAW model, which can simulate the interactive coupling between arc plasma; melting of the electrode; droplet formation, detachment, transfer, and impingement onto the workpiece; and weld pool dynamics. The comprehensive model was used to study the current effect on droplet generation and transfer in the arc [22,23].

For 3D moving GMAW, which has a stronger plasma arc pressure caused by higher current and voltage, the depression of the weld pool by arc pressure should be considered. Furthermore, in moving GMAW, a simultaneous process involving melting of the new solid base metal ahead of the molten pool and solidification of the weld pool on its tail leads to a more complicated mixing process. Modeling on 3D moving GMAW is very limited. Using boundary-fitted coordinates, Kim and Na [24] presented a 3D quasi-steady heat and fluid flow analysis for the moving heat source of the GMAW process with the free surface. However, impingement of droplets, the depression of the weld pool surface by arc pressure, and Marangoni effects were not considered. Ushio and Wu [25] used a boundary-fitted non-orthogonal coordinate system to handle the largely deformed GMA weld pool surface and predicted the area and configuration of weld reinforcement. In their study, the heat delivered by metal transfer was approximated by an internal heat-generation term and the impact of droplets on the weld pool was considered to be a constant force acting on the surface. In other words, the impingement of droplets onto the weld pool was not simulated. Cao et al. [26] used a commercial software package, FLOW-3D, to simulate a transient moving weld pool under the impact of droplet impingement; however, the droplet generation was not considered.

In this paper, the volume of fluid (VOF) technique [27] and the continuum formulation [28] are extended from stationary 2D [20] to 3D moving GMAW. The fluid flow, heat transfer, and species transfer are calculated when droplets carrying mass, momentum, thermal energy, and species periodically impinge onto the weld pool. As in previous studies [12,18], sulfur is selected as the surface-active element which can significantly change the magnitude of surface tension in the weld pool. The VOF technique can handle the transient deformed weld pool surface caused by high-speed droplet impinging onto the free surface and by high arc pressure. The continuum formulation can handle the fusion and solidification, and the liquid region, mushy zone and solid region simultaneously, providing a facility to 
track the moving phase-change boundary. In the present study, as we will focus on weld pool dynamics and the formation of ripples, and in order to save computational time, the droplet generation and the arc plasma are not calculated. Instead, typical droplet conditions, including size, impinging velocity, temperature, and frequency, are assumed based on the previous studies [20-23]. The plasma arc is assumed to be a Gaussian profile. Note these assumptions should not influence the general characteristics of droplet impinging process and the formation of ripples to be analyzed in the present study.

\section{Mathematical Model}

\subsection{Governing Equations}

Fig. 1 is a schematic sketch of a moving GMAW for a plain plate. The 3D $x-y$-z coordinate system is fixed to the stationary base metal, while a $2 \mathrm{D} r-z$ cylindrical coordinate system is moving with the arc center. In GMAW, the total arc energy is split into two parts; one to melt the electrode and generate droplets, and the other to directly heat the base metal. Hence, in addition to thermal energy, mass, and momentum carried by the droplets, arc heat flux is simultaneously impacting on the base metal. Droplets, containing sulfur at different concentrations from that of the base metal, are assumed to periodically impinge onto the base metal in the negative $z$ direction, while they move at the same velocity along the $y$ direction as the arc. For convenience, the mathematical formulation given below is valid for both the base metal and the liquid droplets. However, the temperature, concentration, and impinging velocity are assumed to be constant for the droplets. Once a droplet reaches the free surface, it is immediately considered to be part of the base metal and then the exchanges of momentum, energy, and species between the droplet and the weld pool occur.

The differential equations governing the conservation of mass, momentum, energy and species based on continuum formulation given by Diao and Tsai [28] are modified and employed in the present study and are given below:

Continuity

$$
\frac{\partial}{\partial t}(\rho)+\nabla \cdot(\rho \boldsymbol{V})=0
$$

Momentum

$$
\begin{gathered}
\frac{\partial}{\partial t}(\rho u)+\nabla \cdot(\rho \boldsymbol{V} u)=\nabla \cdot\left(\mu_{l} \frac{\rho}{\rho_{l}} \nabla u\right)-\frac{\partial p}{\partial x}-\frac{\mu_{l}}{K} \frac{\rho}{\rho_{l}}\left(u-u_{s}\right)-\frac{C \rho^{2}}{K^{1 / 2} \rho_{l}}\left|u-u_{s}\right|\left(u-u_{s}\right) \\
-\nabla \cdot\left(\rho f_{s} f_{l} \boldsymbol{V}_{r} u_{r}\right)+\nabla \cdot\left(\mu_{l} u \nabla\left(\frac{\rho}{\rho_{l}}\right)\right)+\boldsymbol{J} \times\left.\boldsymbol{B}\right|_{x}
\end{gathered}
$$




$$
\begin{gathered}
\frac{\partial}{\partial t}(\rho v)+\nabla \cdot(\rho \boldsymbol{V} v)=\nabla \cdot\left(\mu_{l} \frac{\rho}{\rho_{l}} \nabla v\right)-\frac{\partial p}{\partial y}-\frac{\mu_{l}}{K} \frac{\rho}{\rho_{l}}\left(v-v_{s}\right)-\frac{C \rho^{2}}{K^{1 / 2} \rho_{l}}\left|v-v_{s}\right|\left(v-v_{s}\right) \\
-\nabla \cdot\left(\rho f_{s} f_{l} V_{r} v_{r}\right)+\nabla \cdot\left(\mu_{l} v \nabla\left(\frac{\rho}{\rho_{l}}\right)\right)+\boldsymbol{J} \times\left.\boldsymbol{B}\right|_{y} \\
\frac{\partial}{\partial t}(\rho w)+\nabla \cdot(\rho \boldsymbol{V} w)=\rho g+\nabla \cdot\left(\mu_{l} \frac{\rho}{\rho_{l}} \nabla w\right)-\frac{\partial p}{\partial z}-\frac{\mu_{l}}{K} \frac{\rho}{\rho_{l}}\left(w-w_{s}\right)-\frac{C \rho^{2}}{K^{1 / 2} \rho_{l}}\left|w-w_{s}\right|\left(w-w_{s}\right) \\
-\nabla \cdot\left(\rho f_{s} f_{l} \boldsymbol{V}_{r} w_{r}\right)+\nabla \cdot\left(\mu_{l} w \nabla\left(\frac{\rho}{\rho_{l}}\right)\right)+\rho g\left(\beta_{T}\left(T-T_{0}\right)+\beta_{s}\left(f_{l}^{\alpha}-f_{l, 0}^{\alpha}\right)\right)+\boldsymbol{J} \times\left.\boldsymbol{B}\right|_{z}
\end{gathered}
$$

Energy

$$
\frac{\partial}{\partial t}(\rho h)+\nabla \cdot(\rho \boldsymbol{V h})=\nabla \cdot\left(\frac{k}{c_{s}} \nabla h\right)+\nabla \cdot\left(\frac{k}{c_{s}} \nabla\left(h_{s}-h\right)\right)-\nabla \cdot\left(\rho\left(\boldsymbol{V}-\boldsymbol{V}_{s}\right)\left(h_{l}-h\right)\right)
$$

Species

$$
\begin{aligned}
& \frac{\partial}{\partial t}\left(\rho f^{\alpha}\right)+\nabla \cdot\left(\rho \boldsymbol{V} f^{\alpha}\right)=\nabla \cdot\left(\rho D \nabla f^{\alpha}\right)+\nabla \cdot\left(\rho D \nabla\left(f_{l}^{\alpha}-f^{\alpha}\right)\right) \\
& -\nabla \cdot\left(\rho\left(\boldsymbol{V}-\boldsymbol{V}_{s}\right)\left(f_{l}^{\alpha}-f^{\alpha}\right)\right)
\end{aligned}
$$

The above six equations are similar to those given in Ref. [28], except the electromagnetic force (or Lorentz force), $\boldsymbol{J} \times \boldsymbol{B}$, where $\boldsymbol{J}$ is the electric current density vector and $\boldsymbol{B}$ is the magnetic flux vector. As the assumptions to derive the equations and the physical meaning of their terms are given by Diao and Tsai [28], they will not be repeated here. The electromagnetic force is assumed to be independent of the properties of fluid flow in the weld pool, and the $x, y$ and $z$ components will be calculated first, as discussed next, before the velocity is calculated. The second to the last term in Eq. (4) is the buoyancy force which is based on the Boussinesq approximation for natural convection caused by both thermal and solutal convection.

In Eqs. (1)-(6), the continuum density, specific heat, thermal conductivity, mass diffusivity, solid mass fraction, liquid mass fraction, velocity, enthalpy and mass fraction of constitute are defined as follows:

$$
\begin{aligned}
& \rho=g_{s} \rho_{s}+g_{l} \rho_{l} ; \quad c=f_{s} c_{s}+f_{l} c_{l} ; k=g_{s} k_{s}+g_{l} k_{l} \\
& D=f_{s} D_{s}+f_{l} D_{l} ; f_{s}=\frac{g_{s} \rho_{s}}{\rho} ; \quad f_{l}=\frac{g_{l} \rho_{l}}{\rho} \\
& \boldsymbol{V}=f_{s} \boldsymbol{V}_{s}+f_{l} \boldsymbol{V}_{l} ; h=h_{s} f_{s}+h_{l} f_{l} ; f^{\alpha}=f_{s} f_{s}^{\alpha}+f_{l} f_{l}^{\alpha}
\end{aligned}
$$

where $g_{s}$ and $g_{l}$ are the volume fractions of the solid and liquid phases, respectively. If the phase specific heats are assumed constant, the phase enthalpy for the solid and the liquid can be expressed as 


$$
h_{s}=c_{s} T ; \quad h_{l}=c_{l} T+\left(c_{s}-c_{l}\right) T_{s}+H
$$

where $H$ is the latent heat of fusion of the alloy.

The assumption of permeability function in the mushy zone requires the consideration of the growth morphology specific to the alloy under study. In the present study, the permeability function analogous to fluid flow in porous media is assumed employing the Carman-Kozeny equation [29,30],

$$
K=\frac{g_{l}^{3}}{c_{1}\left(1-g_{l}\right)^{2}} ; \quad c_{1}=\frac{180}{d^{2}}
$$

where $d$ is proportional to the dendrite dimension, which is assumed to be a constant and is on the order of $10^{-2} \mathrm{~cm}$. The inertial coefficient, C, can be calculated from [31]

$$
C=0.13 g_{l}^{-3 / 2}
$$

\subsection{Tracking of Solid-Liquid Interface}

The solid/liquid phase-change is handled by the continuum formulation [28]. The third, fourth, and fifth terms in the right-hand-side of Eqs. (2) and (3), and similar terms in Eq. (4), vanish at the solid region because $u=u_{s}=v=v_{s}=w=w_{s}=0$ and $f_{l}=0$ for the solid phase. For the liquid region, since $K$ goes to infinity due to $g_{l}=1$ in Eq. (9) and $f_{s}=0$, all of these terms also vanish. These terms are only valid in the mushy zone where $0<f_{l}<1$ and $0<f_{s}<1$. Therefore, the liquid region, mushy zone and solid region can be handled by the same equations. Also, in GMAW, as the arc heat flux is rather concentrated and the solidification time is very short (as compared to, for example, a casting process), it is expected that the mushy zone in the base metal is very small, and the solid phase velocity is assumed to be zero in the mushy zone. During the fusion and solidification process, latent heat is absorbed or released in the mushy zone. By using enthalpy, conduction in the solid region, conduction and convection in the liquid region and mushy zone, the absorption and release of latent heat are all handled by the same equation, Eq. (5).

\subsection{Tracking of Free Surface}

The algorithm of volume-of-fluid (VOF) is used to track the dynamic geometry of the free surface [27]. The fluid configuration is defined by a volume of fluid function, $F(x, y, z, t)$, which tracks the location of the free surface. This function represents the volume of fluid per unit volume and satisfies the following conservation equation:

$$
\frac{d F}{d t}=\frac{\partial F}{\partial t}+(\boldsymbol{V} \cdot \nabla) F=0
$$


When averaged over the cells of a computing mesh, the average value of $F$ in a cell is equal to the fractional volume of the cell occupied by the fluid. A unit value of $F$ corresponds to a cell full of fluid; whereas, a zero value indicates a cell containing no fluid. Cells with $F$ values between zero and one are partially filled with fluid and identified as surface cells.

\subsection{Boundary Conditions}

The boundary conditions for the solution of Eq. (1) through Eq. (6) are given below:

\subsubsection{Normal to the local free surface}

For cells containing free surface, that is, cells that contain fluid but have one or more empty neighbors, the following pressure condition must be satisfied [27]:

$$
p=p_{v}+\gamma \kappa
$$

where $p$ is the pressure at the free surface in a direction normal to the local free surface, $p_{v}$ is the vapor pressure or any other applied external pressure acting on the free surface, which, in the present study, is the plasma arc pressure. The plasma arc pressure is assumed to have a radial distribution in the moving $r$ $z$ cylindrical coordinate system for the following form [25]:

$$
p_{v}=P_{\max } \exp \left(-\frac{r^{2}}{2 \sigma_{p}^{2}}\right)
$$

where $P_{\max }$ is the maximum arc pressure at the arc center, $r$ is the distance from the arc center $\left(x=x_{a}, y=0\right)$, and $\sigma_{p}$ is the arc pressure distribution parameter. In Eq. (12), $\kappa$ is the free surface curvature given by [27].

$$
\kappa=-\left[\nabla \cdot\left(\frac{\vec{n}}{|\vec{n}|}\right)\right]=\frac{1}{|\vec{n}|}\left[\left(\frac{\vec{n}}{|\vec{n}|} \cdot \nabla\right)|\vec{n}|-(\nabla \cdot \vec{n})\right]
$$

where $\vec{n}$ is a normal vector to the local surface, which is the gradient of VOF function

$$
\vec{n}=\nabla F \text {. }
$$

\subsubsection{Tangential to the local free surface}

The temperature and sulfur concentration-dependent Marangoni shear stress at the free surface in a direction tangential to the local free surface is given by:

$$
\tau_{\vec{s}}=\mu_{l} \frac{\partial(\boldsymbol{V} \cdot \vec{s})}{\partial \vec{n}}=\frac{\partial \gamma}{\partial T} \frac{\partial T}{\partial \vec{s}}+\frac{\partial \gamma}{\partial f^{\alpha}} \frac{\partial f^{\alpha}}{\partial \vec{s}}
$$


where $\vec{s}$ is a tangential vector to the local surface. Surface tension $\gamma$ for a pseudo-binary Fe-S system as a function of temperature, $T$, and the sulfur concentration, $f^{\alpha}$ is given by [32]:

$$
\begin{aligned}
\gamma= & 1.943-4.3 \times 10^{-4}(T-1723) \\
& -R T \times 1.3 \times 10^{-8} \ln \left(1+0.00318 f^{\alpha} \exp \left(\frac{1.66 \times 10^{8}}{R T}\right)\right)
\end{aligned}
$$

where $R$ is the gas constant. Note the surface tension and its gradients as a function of temperature and sulfur concentration for the pseudo-binary Fe-S system are plotted in Ref. [18].

\subsubsection{Top surface}

At the moving arc center, in addition to droplet impingement, arc heat flux is also impacting on the base metal. As arc heat flux is relatively concentrated, it is assumed that the heat flux is perpendicular to the base metal (i.e., neglecting the inclination nature of current and heat flux [20]). Hence, the temperature and concentration boundary conditions at the top surface of the base metal are:

$$
\begin{aligned}
& k \frac{\partial T}{\partial z}=\frac{\eta\left(1-\eta_{d}\right) I u_{w}}{2 \pi \sigma_{q}^{2}} \exp \left(-\frac{r^{2}}{2 \sigma_{q}^{2}}\right)-q_{\text {conv }}-q_{\text {radi }}-q_{\text {evap }} \\
& \frac{\partial f^{\alpha}}{\partial z}=0
\end{aligned}
$$

where $I$ is the welding current, $\eta$ is the arc thermal efficiency, $\eta_{d}$ is the ratio of droplet thermal energy to the total arc energy, $u_{w}$ is the arc voltage, and $\sigma_{q}$ is the arc heat flux distribution parameter. The heat loss due to convection, radiation, and evaporation can be written as:

$$
q_{\text {conv }}=h_{c}\left(T-T_{\infty}\right) ; \quad q_{\text {radi }}=\sigma \varepsilon\left(T^{4}-T_{\infty}^{4}\right) ; \quad q_{\text {evap }}=W H_{v}
$$

where $H_{v}$ is the latent heat for liquid-vapor phase-change and $W$ is the melt mass evaporation rate. For a metal such as steel, $W$ can be written as [33]:

$$
\begin{aligned}
& \log (W)=A_{v}+\log P_{a t m}-0.5 \log T \\
& \log P_{a t m}=6.121-\frac{18836}{T} .
\end{aligned}
$$

2.4.4. Symmetrical $y=0$ plane

$$
\frac{\partial T}{\partial y}=0 ; \frac{\partial u}{\partial y}=0 ; v=0 ; \frac{\partial w}{\partial y}=0 ; \frac{\partial f^{\alpha}}{\partial y}=0 ;
$$

\subsubsection{Other surfaces}

$$
-k \frac{\partial T}{\partial \vec{n}}=q_{\text {conv }} ; \quad u=0 ; \quad v=0 ; \quad w=0 ; \quad \frac{\partial f^{\alpha}}{\partial \vec{n}}=0
$$


where $\vec{n}$ can be x-, y-, or z-direction.

\subsection{Electromagnetic Force}

In each of the Eqs. (2) through (4), there is a term caused by the electromagnetic force that should be calculated first before the calculation of velocity. Assuming the electric field is a quasi-steady-state and the electrical conductivity is constant, the scalar electric potential, $\phi$, satisfies the following Maxwell equation in the local $r$-z coordinate system [20]:

$$
\nabla^{2} \phi=\frac{1}{r} \frac{\partial}{\partial r}\left(r \frac{\partial \phi}{\partial r}\right)+\frac{\partial^{2} \phi}{\partial z^{2}}=0 .
$$

Assuming current is in the negative z-direction, the required boundary conditions for the solution of Eq. (25) are:

$$
\begin{aligned}
& -\sigma_{e} \frac{\partial \phi}{\partial z}=\frac{I}{2 \pi \sigma_{c}^{2}} \exp \left(-\frac{r^{2}}{2 \sigma_{c}^{2}}\right) \text { at the top surface } \\
& \frac{\partial \phi}{\partial z}=0 \text { at } z=0 \\
& \frac{\partial \phi}{\partial r}=0 \text { at } r=0 \\
& \phi=0 \text { at } r=10 \sigma_{c}
\end{aligned}
$$

where $\sigma_{e}$ is the electrical conductivity and $\sigma_{c}$ is the arc current distribution parameter. After the distribution of electrical potential is solved, current density in the $r$ and $z$ directions can be calculated via

$$
J_{r}=-\sigma_{e} \frac{\partial \phi}{\partial r} ; \quad J_{z}=-\sigma_{e} \frac{\partial \phi}{\partial z}
$$

The self-induced azimuthal magnetic field is derived from Ampere’s law through [20]

$$
B_{\theta}=\frac{\mu_{0}}{r} \int_{0}^{r} J_{z} r d r
$$

where $\mu_{0}$ is the magnetic permeability. Finally, the three components of electromagnetic force in Eqs. (2)-(4) are calculated via

$$
\boldsymbol{J} \times\left.\boldsymbol{B}\right|_{x}=-B_{\theta} J_{z} \frac{x-x_{a}}{r} ; \quad \boldsymbol{J} \times\left.\boldsymbol{B}\right|_{y}=-B_{\theta} J_{z} \frac{y}{r} ; \quad \boldsymbol{J} \times\left.\boldsymbol{B}\right|_{z}=B_{\theta} J_{r}
$$

\section{Numerical Considerations}

Finite difference conventions follow that of the MAC scheme [34] in which $x$-, $y$ - and $z$-velocity components are located at cell face centers on lines of constant $x, y$ and $z$, respectively, and the pressure, 
VOF function, temperature, and concentration are located at cell centers. The numerical procedures used in the present study for the aforementioned governing differential equations are briefly discussed in the following.

1. The momentum differential equations are cast into the general format suggested by Patankar [35]:

$$
\frac{\partial(\rho \boldsymbol{V})}{\partial t}+\nabla \cdot(\rho \boldsymbol{V})=\nabla \cdot \boldsymbol{\tau}+S_{\phi}
$$

where $\boldsymbol{\tau}$ is the viscous stress tensor and $S_{\phi}$ is the source term, which includes the pressure gradient, Darcy's function, the relative phase motion, electromagnetic force, gravitational force, and buoyancy force in the momentum equations. Eq. (33) is solved in finite difference form with a two-step projection method involving the time discretization of momentum Eqs. (2)-(4). The step one equation is

$$
\frac{\tilde{\boldsymbol{V}}-\boldsymbol{V}^{n}}{\delta t}=-\nabla \cdot(\boldsymbol{V} \boldsymbol{V})^{n}+\frac{1}{\rho^{n}} \nabla \cdot \boldsymbol{\tau}^{n}+\frac{1}{\rho^{n}} S_{\phi}^{n}
$$

where the velocity field $\tilde{\boldsymbol{V}}$ is explicitly computed from incremental changes in the field $\boldsymbol{V}^{\boldsymbol{n}}$ resulting from advection, viscosity, and the source term. In step two, the velocity field $\tilde{\boldsymbol{V}}$ is projected onto a zero-divergence vector field $\boldsymbol{V}^{n+1}$ using the following two equations:

$$
\begin{aligned}
& \frac{\boldsymbol{V}^{n+1}-\tilde{\boldsymbol{V}}}{\delta t}=-\frac{1}{\rho^{n}} \nabla p^{n+1} \\
& \nabla \cdot V^{n+1}=0 .
\end{aligned}
$$

These two equations can be combined into a single Poisson equation for the pressure, which is solved by the incomplete Cholesky conjugate gradient (ICCG) solution technique [36]:

$$
\nabla \cdot\left[\frac{1}{\rho^{n}} \nabla p^{n+1}\right]=\frac{\nabla \cdot \tilde{\boldsymbol{V}}}{\delta t} .
$$

2. Eq. (11) is initiated by defining an immediate $\tilde{F}$ :

$$
\tilde{F}=F^{n}-\delta t \nabla \cdot\left(\boldsymbol{V} F^{n}\right) .
$$

It can be completed with a divergence correction to get $F^{n+1}$ for the new fluid domain:

$$
F^{n+1}=\tilde{F}+\delta t(\nabla \cdot V) F^{n} .
$$

3. The equations of energy, species, and Maxwell are solved explicitly to determine the distribution of temperature, concentration, and electromagnetic field, respectively. Eq. (7) is then used to update the liquid-fraction, material properties, etc. 
4. Finally, at a certain period of time, a new droplet with given diameter, temperature, and concentration is produced and the surface boundary condition is imposed on this new droplet. Repetition of the aforementioned steps produces the solution until the time is out.

Since the governing equations are valid in the regions of liquid, solid and mushy zone, there is no need to track the geometrical shape and the extent of each region. Hence, a fixed-grid system is used in the numerical calculation. As the dimensions of the ripple are relatively small, a great deal of effort was exercised to achieve accurate and consistent results. After some extensive tests, a grid system of $408 \times 102 \times 62$ points is selected for the total computational domain of $300 \mathrm{~mm} \times 30 \mathrm{~mm} \times 0.9 \mathrm{~mm}$. Due to the symmetry of the $x$-z plane of the domain, only half of the grid points $(408 \times 51 \times 62)$ are used in the actual calculation. As the weld pool moves in the welding direction, an adaptive grid system is employed, having finer grids in the weld pool. The finer grids concentrating on and around the weld pool move with the weld pool as the welding proceeds. However, in order to enhance readability, only partial of the grid points are used for all velocity plots that will be presented below.

Calculations were executed on the HP-9000/C200 workstations, and it took 24 hours of CPU time to simulate about 1.50 seconds of real-time welding. The average time step is $10^{-4}$ seconds and the smallest time step, about $10^{-5}$ seconds, occurs when the droplet impinges onto the weld pool.

\section{Results and Discussion}

The flow pattern, distributions of temperature and sulfur concentration, and the final weld pool penetration for a moving GMAW for 304 stainless steels were calculated. In order to simulate a realistic welding process, typical welding conditions were selected as a forced input. As in previous studies $[12,18]$, to examine the effect of surface tension on weld pool fluid flow, sulfur was selected as the surface active element. The base metal is assumed to contain $100 \mathrm{ppm}$ of sulfur, while the droplets contain $300 \mathrm{ppm}$. The welding conditions and material properties of both base metal and liquid droplets used in the computation are summarized in Table 1. The droplet is assumed to be spherical, and based on the welding conditions and the results from our previous studies on droplets generation [20-23], the following droplet conditions are selected in the present study: droplet diameter $(1.5 \mathrm{~mm})$, droplet generation frequency $(65 \mathrm{~Hz})$, droplet impinging velocity $(70 \mathrm{~cm} / \mathrm{s})$, and droplet temperature $(2500 \mathrm{~K})$.

To avoid end effects, simulation was started when the first droplet impinged onto the base metal at $\mathrm{X}$ $=10 \mathrm{~mm}$. There were 65 droplets per second falling onto the base metal in the negative $\mathrm{z}$ direction. At the same time, the droplet had the same speed $(7 \mathrm{~mm} / \mathrm{s})$ as the welding in the $\mathrm{x}$ direction. Fig. 2 is a partial view of a three-dimensional mesh system and weld deposition at $t=2.564 \mathrm{~s}$. As indicated from the figure, the weld bead begins at about $\mathrm{X}=7.0 \mathrm{~mm}$ because when the droplets impinge onto the base metal, part of 
the metal "flows" outward creating a skirt effect. The maximum deposition height is about $1.5 \mathrm{~mm}$. There are some distinct ripples in the solidified weld puddle, two moving "waves" in the molten weld pool and a “crater" caused by the impinging droplet at that instant of time. In the following sections, temperature, fluid flow, and sulfur distributions in the weld pool during the welding process will be discussed first. The mechanisms leading to the formation of ripples will then be explained in detail.

\subsection{Interactions Between Filler Droplets and Weld Pool}

The numerical simulation begins when the plasma arc is turned on and the first droplet is assumed to generate immediately and ends when the plasma arc is turned off and the last droplet falls. Transient weld pool shape and distributions of temperature, velocity, and sulfur concentration are calculated at each time step. Hence, tremendous data have been obtained during simulation. However, only some representative results are selected and presented below to explain typical, common phenomena during the welding process.

\subsubsection{Side view of the impinging process}

Fig. 3 is the side-view (at $\mathrm{Y}=0$ ) showing the sequence of a droplet impinging process onto the weld pool during one typical periodic impinging process and the distributions of temperature at different times. Figs. 4 and 5 are, respectively, the corresponding distributions of velocity and sulfur concentration in the weld pool.

At $t=2.938 \mathrm{~s}$, a new droplet is ready to impinge onto the weld pool at the arc center, Fig. 3 . The droplet has a vertical speed about $-70 \mathrm{~cm} / \mathrm{s}$ and a horizontal speed $7 \mathrm{~mm} / \mathrm{s}$. The isotherms of the liquidus line at $1725 \mathrm{~K}$ and the solidus line at $1670 \mathrm{~K}$ are shown in the figure. As shown, the shape of the isotherms in the liquid region is more irregular, as compared to that in the solid phase. There are two "hot spots” in the weld pool; one spot is near the arc center ( $\mathrm{X}$ is about $30 \mathrm{~mm}$ ) which extends downward to the bottom of the weld pool. This hot spot represents the previous droplet, but is skewed toward the lefthand-side of the weld pool. The other hot spot is located at about X $=25 \mathrm{~mm}$ and near the surface of the weld pool and at its highest level. At the solidified portion of the weld bead ( $\mathrm{X}$ is about $20 \mathrm{~mm}$ ), several ripples are clearly seen, which will be discussed next. The corresponding velocity distribution in Fig. 4 can be used to explain the complicated temperature distribution at $\mathrm{t}=2.938 \mathrm{~s}$.

In Fig. 4, $\mathrm{t}=2.938 \mathrm{~s}$, resulting from the previous droplet, the fluid flows outward in two directions at about $\mathrm{X}=33 \mathrm{~mm}$, one along the top of the base metal in the welding direction and the other toward the left-hand side at a much higher speed. The left-hand fluid is downward and along the inclined solid-phase base metal, and then flows upward after reaching the bottom of the weld pool. This creates a clockwise vortex between $X=28 \mathrm{~mm}$ and $X=32 \mathrm{~mm}$. As the former droplet carries higher thermal energy, a 
skewed hot spot is formed in the weld pool near the arc center. It is noted that the flow of hightemperature fluid downward and toward the left-hand side has a "cutting" effect to melt the base metal so that the weld pool "moves" to the right-hand side.

The aforementioned upward "hot fluid" turns left near the weld pool surface and flows to the lefthand side (between $X=25.0 \mathrm{~mm}$ and $\mathrm{X}=30.0 \mathrm{~mm}$ ) and collides with another fluid from the left-hand side of the weld pool, leading to another "hot spot" near the surface. It is noted that, in addition to the thermal energy carried by droplets, thermal arc flux also impacts on the surface of the weld pool, resulting in a relatively high surface temperature near the arc center. The fluid collision creates a "bump" with the highest liquid level at about $\mathrm{X}=25 \mathrm{~mm}$, Fig. 3. There are three visible vortexes in the weld pool interacting with each other.

The surface fluid between $X=25.0 \mathrm{~mm}$ and $\mathrm{X}=30.0 \mathrm{~mm}$ flows outward away from the arc center. Surface flow in a weld pool is attributed mainly to the combined effect of three forces: 1) the arc pressure, which produces an outward force at the surface close to the arc center; 2) the impinging force, which creates an outer flow at the surface surrounding the droplet (see Fig. 4 at $t=2.942 \mathrm{~s}$ ); and 3) the surface tension, which is a function of temperature and sulfur concentration. The weld pool surface temperature between the arc center and $\mathrm{X}=25.0 \mathrm{~mm}$ is above $2300 \mathrm{~K}$, Fig. 3, and the surface sulfur concentration is near 300 ppm, Fig. 5. According to Eq. (17), the temperature coefficient of surface tension is negative, i.e., fluid flows from a location at higher temperature to a location at lower temperature. Therefore, the Marangoni force is outward from the arc center. Hence, all three aforementioned forces lead to an outflow of surface fluid away from the arc center.

At the same time, surface fluid between the tail edge of the weld pool and $\mathrm{X}=24.0 \mathrm{~mm}$ flows toward the arc center. As the surface temperature is below $2000 \mathrm{~K}$, Fig. 3, and surface sulfur concentration is above 250 ppm, Fig. 5, the temperature coefficient of surface tension is positive, Eq. (17). Accordingly, the Marangoni force is toward the arc center, leading to a collision with the aforementioned surface flow from the arc center at about $X=25.0 \mathrm{~mm}$. As a result, two vortexes are formed; one near the edge of the weld pool being clockwise, and the other being counter-clockwise carrying high-temperature surface fluid downward near $X=25.0 \mathrm{~mm}$. As shown in Fig. 3, the two hot spots, as discussed above, form the shape of "skewed W", which moves as the welding proceeds in the right-hand direction. The shape of the liquidus line implies that at a location along the horizontal direction (say $\mathrm{Z}=5.5 \mathrm{~mm}$ ) the weld subjects to a cycle of high temperature (possibly melted), low temperature (possibly re-solidified), high temperature (possibly re-melted), and low temperature (final solidification) as welding moves in the right-hand direction. This heat-cool phenomenon can be important if one wants to consider weld microstructure evolution and residue stress in the weld. 
At $t=2.942 \mathrm{~s}$, the droplet has already plunged into the weld pool at the arc center. The just flattened surface is deformed by the impinging momentum, while the temperature, Fig. 3, and sulfur concentration, Fig. 5, near the arc center are higher than those of its surroundings. The impinging momentum destroys the existing vortex under the arc center and enhances the outer flow at the surface near the arc center (between $X=25.0 \mathrm{~mm}$ and $X=30.0 \mathrm{~mm}$ ), Fig. 4. The other two existing vortexes still exist.

At $\mathrm{t}=2.946 \mathrm{~s}$ and $\mathrm{t}=2.954 \mathrm{~s}$, the flow patterns are similar to that at $\mathrm{t}=2.942 \mathrm{~s}$. However, the "crater" becomes deeper and wider, and the surface fluid velocity near the left-hand side of the crater increases, pushing the liquid level there upward. As a result, part of the kinetic energy of the droplet has converted into the potential energy of the weld pool fluid. The formation of a deep crater can lead to three important effects: 1) the subsequent droplets can easily bring in thermal energy to the bottom of the weld pool; 2) the arc heat flux can easily reach the bottom of the weld pool; and 3) the impinging droplet can mix directly with the just-melted base metal, which may have different compositions from the droplet. Overall, the formation of a crater facilitates the cutting effect on base metal, influencing weld penetration and weld mixing.

Between $\mathrm{t}=2.954 \mathrm{~s}$ and $\mathrm{t}=2.2992 \mathrm{~s}$, there are three droplets hitting the base metal before the crater is closed up. It is seen in Fig. 4, at $t=2.954 \mathrm{~s}, 2.961 \mathrm{~s}$, and $2.977 \mathrm{~s}$, the flow surrounding the crater is outward (i.e., the crater is expanding), while at $\mathrm{t}=2.969$, $2.984 \mathrm{~s}$, and $2.992 \mathrm{~s}$, the flow surrounding the crater is inward (i.e., the crater is closing up). Hence, a crater is formed by droplet impingement, and the crater tends to close itself up due to hydrostatic force. However, because of the continued impingement by subsequent droplets, there is no chance for the crater created by the droplet at $t=2.938$ to close up until $t$ $=3.000 \mathrm{~s}$, Fig. 3. It is seen that the distributions of temperature, velocity, and concentration at $\mathrm{t}=3.000 \mathrm{~s}$ are very similar to the corresponding figures at $\mathrm{t}=2.938 \mathrm{~s}$. Hence, for the welding conditions used in the present study, it takes about 4 droplets to complete a "cycle" for the transport phenomena occurring in the weld pool. The frequency of opening and closing-up of the crater depends mainly on the droplet size, momentum, temperature, drop frequency, and arc flux energy.

\subsubsection{Front view of the impinging process}

Fig. 6 is the front view of the cross-sectional temperature distribution at different positions along the arc moving direction at $t=2.938$ s. Figs. 7 and 8 are, respectively, the corresponding velocity and sulfur concentration distribution in the weld pool.

At $\mathrm{X}=34.77 \mathrm{~mm}, 4.2 \mathrm{~mm}$ ahead of the arc center, the base metal is already heated by the arc energy, but still in the solid state with initial sulfur concentration. The maximum temperature occurs at the center ( $\mathrm{Y}=0$ ), as expected. At $\mathrm{X}=30.57 \mathrm{~mm}$, which is the arc center, a droplet with a temperature of $2500 \mathrm{~K}$ and sulfur concentration of $300 \mathrm{ppm}$ appears just above the pool and is ready to impinge onto the weld 
pool. The small indent at the arc center is caused by the previous droplet, and the highest liquid level on each side of the weld pool (symmetry) is part of the "wave” propagating outward from the arc center, as shown in Fig. 9. As the fluid tends to flatten the crater created by the previous droplet, the fluid surrounding the arc center flows inward and collides at the arc center, leading to an upward flow, Fig. 7. Due to mixing with the previous downward droplet and the action of downward electromagnetic force, the fluid flow close to the bottom is downward, creating a weak counter-clockwise vortex. At a distance of about $2 \mathrm{~mm}$ from the arc center, the temperature is above $2300 \mathrm{~K}$, Fig. 6, and the concentration is below 250 ppm, Fig. 8. Hence, according to Eq. (17), there is a negative surface tension gradient leading to an outer flow away from the arc center, Fig. 7. This outward flow at a distance of about $2 \mathrm{~mm}$ from the arc center is enhanced by the fact that fluid at a higher location tends to flow to a lower location under the action of hydrostatic force. At $\mathrm{X}=30.57 \mathrm{~mm}$, the melted region of base metal and weld penetration are small and, as a result, the mixing between droplets and base metal is very limited, Fig. 8.

At $X=27.57 \mathrm{~mm}$, which is $3 \mathrm{~mm}$ behind the arc center, the two highest liquid levels correspond to the moving wave, as shown in Fig. 9. There are two clockwise vortexes near the arc center, Fig. 7. The clockwise vortexes at $\mathrm{X}=30.57 \mathrm{~mm}$ and the counter-clockwise vortexes at $\mathrm{X}=27.57 \mathrm{~mm}$, and their interactions can be seen in Fig. 4. Fig. 8 shows at $X=27.57 \mathrm{~mm}$ the melted region of base metal becoming wider and deeper, and the on-going mixing appears vigorous. At $X=30.57 \mathrm{~mm}$, the "bumps" near the edge of the weld pool belong to the same wave, as shown in Fig. 9, as those appearing at 27.57 $\mathrm{mm}$ and $25.77 \mathrm{~mm}$, in which there is only one bump in the center.

At $X=20.07 \mathrm{~mm}$, about $0.5 \mathrm{~mm}$ behind the liquid weld pool, the weld is fully solidified and is the final shape of the weld bead. As expected, the highest temperature occurs at the center, Fig. 6. It is seen from Fig. 8, although the sulfur concentration near the top center of the weld bead is higher, the sulfur concentration distribution in the solidified weld is fairly uniform (less than $3 \%$ variation) except near the skirt of the weld bead. The very high sulfur concentration near the skirt of the weld bead is caused by the fact that very poor mixing between the base metal and the droplets near the skirt and the droplet containing high sulfur is solidified at the skirt of the bead.

\subsubsection{Three-dimensional and top view of the impinging process}

Fig. 9 is a partial view of the three-dimensional mesh system, weld bead shape, and velocity distribution at $t=2.938$ s. Fig. 10 shows the corresponding top view of velocity, temperature, and sulfur concentration distributions. The shape of the crater is clearly seen in Fig. 9. Next to the crater, there is a "wave" propagating away from the arc center in the negative x-direction. This wave corresponds to the "bumps" discussed in the previous cross-sectional view in Fig. 6. Outside the weld pool, there are four

distinct ripples at the surface of the solidified weld bead. The weld pool is wider at the front part of the 
weld pool and gradually becomes narrower toward the rear part of the pool, since the weld pool is losing heat through conduction and convection to the base metal, and through convection and radiation to the surroundings. Hence, the weld pool has a typical “chevron” shape, as frequently reported in literature.

The top-view velocity distribution is very complicated, especially for areas in and near the crater. To visualize the velocity field in the weld pool, it would be helpful to cross-reference Figs. 4, 7, and 9. The surface fluid in the area, $30.57 \mathrm{~mm}<\mathrm{X}<32 \mathrm{~mm}$ and $-2 \mathrm{~mm}<\mathrm{Y}<2 \mathrm{~mm}$, flows toward the arc center in order to flatten the crater created by the previous droplet. The surface fluid between the arc center (at $\mathrm{X}=$ $30.57 \mathrm{~mm}$ ) and the wave flows outward, and the surface fluid behind $\mathrm{X}=26 \mathrm{~mm}$ flows toward the arc center. All these surface fluid flows are caused mainly by surface tension force (Marangoni force), which has been previously discussed. The fluid near the front edge of the weld pool has a slow velocity and is forward in the same arc moving direction.

Fig. 10 shows that the temperature near the arc center is higher and decreases toward the tail direction of the weld pool. As expected, the sulfur concentration distribution is also very complex in the weld pool. The mixing of sulfur in the weld pool is caused by the combined effect of droplet impingement, surface tension, gravity, electromagnetic force, and arc pressure. There is a high sulfur concentration near the center of the weld pool, which is caused by the upturn of the droplet after impinging onto the weld pool, as discussed in Figs. 3, 4, and 5. The two "white spots" in the weld pool shown in Fig. 10 correspond to the bottom of the crater, as shown in Fig. 5. However, the sulfur concentration in the solidified weld bead is fairly uniform overall, as shown in Fig. 8.

\subsection{The Dynamic Formation of Ripples}

Ripples left in the solidified weld bead, as shown in Fig. 2, are very common in gas metal arc welding. Understanding the mechanisms leading to the formation of ripples will be helpful in determining the weld bead shape, including undercut and overlap which can affect weld quality. In the following, efforts will be devoted to explaining the formation of ripples using the previous figures. Fig. 3 will be used to discuss a periodic process to "generate" one ripple in the final weld bead.

At $\mathrm{t}=2.938 \mathrm{~s}$ in Fig. 3 on the right-hand side, there are four ripples that have been formed (i.e., solidified) while the liquid level of the weld pool next to the ripples is as high as that of the "tip" of the ripples. It is noted that the isotherm $1725 \mathrm{~K}$ shows the liquidus line, while the curve of $1670 \mathrm{~K}$ indicates the solidus line. As GMAW generally involves a highly concentrated heat flux, the mushy zone (between the liquidus line and the solidus line) is very small and has nearly no flow, as seen in Figs. 3 and 4 . This is consistent with the assumption made previously in the mathematical model. As seen at $t=2.938 \mathrm{~s}$, the 
liquidus line reaches nearly the same height as that of the tip of the ripples. As time passes, filler droplets continue to "bomb" the weld pool, creating a crater.

By the conservation of mass, the formation of the crater will "push" the overall fluid level of the weld pool upward, including the propagating waves. As explained previously, the droplet impingement is at such a frequency (65 droplets per second in the present study) that the size of the crater increases until $\mathrm{t}=$ $2.961 \mathrm{~s}$, Fig. 3. At this time, some of the weld pool liquid starts to "fill-up" the crater, and the size of the crater decreases. As a result, the fluid level falls to a height which is the same as that of the "valley" of the ripples, as shown at $\mathrm{t}=2.969 \mathrm{~s}$. As droplets continue to impinge onto the weld pool, they also move at a speed of $7 \mathrm{~mm} / \mathrm{s}$ in the welding direction. At the same time, solidification continues to "move" from the left-hand side in Fig. 3 to the right, as evidenced from the movement of both the liquidus and the solidus lines. Hence, the high-level liquid next to the last ripple, as shown at $t=2.938 \mathrm{~s}$ through $2.9556 \mathrm{~s}$ is "frozen" while the rest of the liquid falls.

A new ripple starts to form at $t=2.969 \mathrm{~s}$ as the liquid level in the weld pool decreases. The ripple formation is almost complete, i.e., without further changing the shape at $t=2.984$. At this instant of time, the liquid level at the tail edge is at its lowest and a new ripple is fully developed. It is noted that the lowest liquid level near the tail edge of the weld pool does not correspond to the instant when the crater is smallest or completely filled up. In other words, the weld pool liquid level is influenced by the size of the crater and the wave propagating in the weld pool. There is a time lag between the droplet impingement, generation of wave, and the location and height of the propagating wave. At $t=2.977 \mathrm{~s}$, the weld pool is almost flat, although a large crater still exists, but a new high-level fluid begins to build at $\mathrm{t}=2.984 \mathrm{~s}$.

At $t=2.984 \mathrm{~s}$, the liquid level between the tail edge and the arc center begins to increase and propagate outward. This is the beginning of the process to form another new ripple. At $t=2.992 \mathrm{~s}$ and $3.000 \mathrm{~s}$, the liquid level at the tail edge is still increasing. At $\mathrm{t}=3.000 \mathrm{~s}$, the weld pool shape, temperature distribution, and flow pattern all are very similar to those at $\mathrm{t}=2.938 \mathrm{~s}$. This is approximately one cycle for the formation of a ripple. It is very interesting to observe in Fig. 3 that the wave never reaches the tail edge of the weld pool but stops in the middle. However, the moving wave carries momentum that helps to raise the liquid level near the tail edge.

Hence, the mechanisms leading to the formation of a ripple can be summarized as follows. First, due to droplet impingement, a crater is created pushing the fluid upward and away from the arc center. A wave with its peak height greater than the liquid level is also generated, propagating outward. Second, due to hydrostatic force, the high-level fluid tends to fill-up the crater and decreases the liquid level. Third, as welding proceeds in the right-hand direction, solidification also moves in the same direction. As high-level fluid near the tail edge is solidified before moving down and flowing back in the crater direction, a ripple is created. Hence, the formation of ripples is related to the open and close-up of the 
crater and the resulting up-and-down of the weld pool fluid level. The time required for the up-and-down cycle for fluid level near the tail edge and the "pitch" of the ripples depends on many welding parameters, including droplet size (electrode diameter), droplet momentum, drop frequency (wire feed speed), welding speed, welding power (current and voltage), and others. It is noted that all these welding parameters and conditions are coupled together.

Since the formation of a ripple is closely related to the dynamic behavior of a crater, it is interesting to find that the life cycle of a crater (i.e., from open to close-up) is approximately the same as creating a ripple. The distance between two ripples in the final bead is approximately $0.45 \mathrm{~mm}$. The welding speed is $7.0 \mathrm{~mm} / \mathrm{s}$, therefore the time to produce one ripple is about $0.064 \mathrm{~s}$. Since the drop frequency is 65 droplets per second, about 4 droplets have impinged onto the weld pool during this period of time. Also, from $t=2.938 \mathrm{~s}$ to $t=3.000 \mathrm{~s}$, the time difference is $0.062 \mathrm{~s}$. It takes approximately $0.001 \mathrm{~s}$ more for the crater at $\mathrm{t}=3.000 \mathrm{~s}$ to become similar to that at $\mathrm{t}=2.938 \mathrm{~s}$. Hence, the time required to create a crater and later to fill-up the crater is about $0.063 \mathrm{~s}$, which is very close to the time required to produce one ripple.

\section{Conclusions}

The fluid flow and heat and mass transfer in the weld pool for a moving GMAW were analyzed using the VOF technique and the continuum formulation. In the modeling, the droplet impingement, electromagnetic force, plasma arc force, and Marangoni effect were all considered. Transient weld pool shape and temperature, velocity, and species distributions were calculated. A crater was opened as a result of droplet impingement, and its close-up was caused mainly by hydrostatic force. The crater's open and close-up led to the "up-and-down" of the fluid level in the weld pool. A ripple was formed as solidification proceeds in the welding direction. Hence, the periodic impingement of droplets, weld pool dynamics, and solidification all contributed to the formation of ripples. Due to strong mixing between droplets and the weld pool, species concentration distribution was fairly uniform in the weld pool for a moving GMAW. The modeling developed in the present study laid the required foundation to conduct parametric studies for understanding the effects of various welding parameters on the shape of the weld bead and weld quality.

\section{Acknowledgment}

The work was supported by the U.S. Army Research Office under grant number DAAH04-95-10136 and the General Motors Corporation, which is gratefully acknowledged.

\section{References}


[1] E. F. Nippes et al, Welding, brazing, and soldering, Metals Handbook (Ninth Edition), volume 6, American Society for Metals (1983) 153-181.

[2] J. Szekely, Transport phenomena in welds with emphasis on free surface phenomena, in Recent Trends in Welding Science and Technology, TWR'89, edited by S. A. David and J. M. Vitek, ASM International (1990) 3-10.

[3] P. Dutta, Y. Joshi, and R. Janaswami, Thermal modelling of GTAW process with nonaxisymmetric boundary conditions, Numer. Heat Transfer A 27 (1995) 499-518.

[4] N. Chakraborty, S. Chakraborty, and P. Dutta, Three-dimensional modeling of turbulent weld pool convection in GTAW processes, Numer. Heat Transfer A 45 (2004) 391-413.

[5] A.A. Reddy, B. Guha, D.R.G. Achar, Finite element modeling of three-dimensional transient heat transfer in stainless steel (304) pulsed GTA weldments, Numer. Heat Transfer A 41 (2002) 41-64.

[6] W.H. Kim, H.G. Fan, S.J. Na, Effect of various driving forces on heat and mass transfer in arc welding, Numer. Heat Transfer A 32 (1997) 633-652.

[7] G.M. Oreper and J. Szekely, Heat and fluid-flow phenomena in weld pools, J. Fluid Mech. 147 (1984) 53-79.

[8] M. Kanouff and R. Greif, The unsteady development of a GTA weld pool, Int. J. Heat Mass Transfer 35 (1992) 967-979.

[9] R.-H. Yeh, S.-P. Liaw, Y.-P. Tu, Transient three-dimensional analysis of gas tungsten arc welding plates, Numer. Heat Transfer A 51 (2007) 573-592.

[10] G.M. Oreper, T.W. Eagar and J. Szekely, Convection in arc weld pools, Welding J. (1983) 307s312s.

[11] R.T.C. Choo and J. Szekely, The effect of gas shear stress on Marangoni flows in arc welding, Welding J. (1991) 223s-233s.

[12] M.C. Tsai and S. Kou, Electromagnetic-force-induced convection in weld pools with a free surface, Welding J. (1990) 241s-246s.

[13] Y. Wang, Q. Shi and H.L. Tsai, Modeling of the effects of surface-active elements on flow patterns and weld penetration, Metall. Trans. 32B (2001) 145-161.

[14] M.C. Tsao and C.S. Wu, Fluid flow and heat transfer in GMA weld pools, Welding J. (1988) 70s$75 \mathrm{~s}$.

[15] J. Jaidi and P. Dutta, Modeling of transport phenomena in a gas metal arc welding process, Numer. Heat Transfer A 40 (2001) 543-562.

[16] Y. Wang and H.L. Tsai, Impingement of filler droplets and weld pool dynamics during gas metal arc welding process, Int. J. Heat Mass Transfer 44 (2001) 2067-2080. 
[17] H.G. Fan and R. Kovacevic, A unified model of transport phenomena in gas metal arc welding including electrode, arc plasma and molten pool, J. Phys. D: Appl. Phys. 37 (2004) 2531-2544.

[18] H.G. Fan and R. Kovacevic, Droplet formation, detachment, and impingement on the molten pool in gas metal arc welding, Metall. Trans. 30B (1999) 791-801.

[19] Y. Wang and H.L. Tsai, Effects of surface active elements on weld pool fluid flow and weld penetration in gas metal arc welding, Metall. Trans. 32B (2001) 501-515.

[20] F.L. Zhu, H.L. Tsai, S.P. Marin and P.C. Wang, A comprehensive model on the transport phenomena during gas metal arc welding process, Prog. Comput. Fluid Dyn. 4 (2) (2004) 99-117.

[21] J. Hu and H.L. Tsai, Heat and mass transfer in gas metal arc welding, Part I: The arc, Int. J. Heat Mass Transfer 50 (2007) 833-846.

[22] J. Hu and H.L. Tsai, Heat and mass transfer in gas metal arc welding, Part II: The metal, Int. J. Heat Mass Transfer 50 (2007) 808-820.

[23] J. Hu and H.L. Tsai, Effects of current on droplet generation and arc plasma in gas metal arc welding, J. Appl. Phys. 100 (2006) 053304.

[24] J. Hu and H.L. Tsai, Metal transfer and arc plasma in gas metal arc welding, ASME J. Heat Transfer, 129 (2007) 1025-1035.

[25] J.W. Kim and S.J. Na, A study on the three-dimensional analysis of heat and fluid flow in gas metal arc welding using boundary-fitted coordinates, ASME J. Eng. Indus. 116 (1994) 78-85.

[26] M. Ushio and C.S. Wu, Mathematical modeling of three-dimensional heat and fluid flow in a moving gas metal arc weld pool, Metall. Trans. 28B (1997) 509-516.

[27] Z. Cao, Z. Yang and X.L. Chen, Three-dimensional simulation of transient GMA weld pool with free surface, Welding J. (2004) 169-176s.

[28] D.B. Kothe and R.C. Mjolsness, Ripple: a new model for incompressible flows with free surfaces, LA-UR-91-2818, Los Alamos National Laboratory, 1991.

[29] Q.Z. Diao and H.L. Tsai, Modeling of solute redistribution in the mushy zone during solidification of aluminum-copper alloys, Metall. Trans. 24A (1993) 963-973.

[30] P.C. Carman, Fluid flow through granular beds, Trans. Inst. Chem. Eng. 15 (1937) 150-166.

[31] K. Kubo and R.D. Pehlke, Mathematical modeling of porosity formation in solidification, Metall. Trans. 16A (1985) 823-829.

[32] G.S. Beavers and E.M. Sparrow, Non-Darcy flow through fibrous porous media, J. Appl. Mech. 36 (1969) $711-714$.

[33] P. Sahoo, T. DeBroy and M.J. Mcnallan, Surface tension of binary metal-surface active solute systems under conditions relevant to welding metallurgy, Metall. Trans. 19B (1988) 483-491. 
[34] T. Zacharia, S.A. David and J.M. Vitek, Effect of evaporation and temperature-dependent material properties on weld pool development, Metall. Trans. 22B (1992) 233-241.

[35] J.E. Welch, F.H. Harlow, J.P. Shannon and B.J. Daly, The MAC Method: A computing technique for solving viscous, incompressible, transient fluid-flow problems involving free surface, LA-3425, Los Alamos Scientific Laboratory, 1966.

[36] S.V. Patankar, Numerical Heat Transfer and Fluid Flow, Hemisphere, New York, 1980.

[37] D.S. Kerhaw, The incomplete Cholesky-Conjugate Gradient Method for the interactive solution of systems of linear equations, J. Comput. Phys. 26 (1978) 43-65. 
Table 1. Thermophysical properties of 304 stainless steel and welding conditions

Nomenclature Symbol

Value (unit)

Constant in Eq. (21)

$A_{v}$

2.52

Specific heat of solid phase

$$
C_{S}
$$

Specific heat of liquid phase

Mass diffusion coefficient of solid phase

Mass diffusion coefficient of liquid phase

Thermal conductivity of solid phase

Thermal conductivity of liquid phase

Density of solid phase

Density of liquid phase

Thermal expansion coefficient

Solutal expansion coefficient

Radiation emissivity

Dynamic viscosity

Latent heat of fusion

Magnetic permeability

Solidus temperature

Liquidus temperature

Reference temperature

Ambient temperature

Convective heat transfer coefficient

Latent heat of vaporization

$\begin{array}{ll}A_{v} & 2.52 \\ c_{s} & 700\left(\mathrm{~J} \mathrm{~kg}^{-1} \mathrm{~K}^{-1}\right) \\ c_{l} & 780\left(\mathrm{~J} \mathrm{~kg}^{-1} \mathrm{~K}^{-1}\right) \\ D_{s} & \cong 0 \\ D_{l} & 3 \times 10^{-5}\left(\mathrm{~cm}^{2} \mathrm{~s}^{-1}\right) \\ k_{s} & 22\left(\mathrm{~W} \mathrm{~m}^{-1} \mathrm{~K}^{-1}\right) \\ k_{l} & 22\left(\mathrm{~W} \mathrm{~m}^{-1} \mathrm{~K}^{-1}\right) \\ \rho_{s} & 7200\left(\mathrm{~kg} \mathrm{~m}^{-3}\right) \\ \rho_{l} & 6900\left(\mathrm{~kg} \mathrm{~m}^{-3}\right) \\ \beta_{T} & 4.95 \times 10^{-5}\left(\mathrm{~K}^{-1}\right) \\ \beta_{s} & -2.0 \\ \varepsilon & 0.4 \\ \mu_{l} & 0.006\left(\mathrm{~kg} \mathrm{~m}^{-1} \mathrm{~s}^{-1}\right) \\ H & 2.47 \times 10^{5}\left(\mathrm{~J} \mathrm{~kg}^{-1}\right) \\ \mu_{0} & 1.26 \times 10^{-6}\left(\mathrm{H} \mathrm{m}^{-1}\right) \\ T_{s} & 1670(\mathrm{~K}) \\ T_{l} & 1725(\mathrm{~K}) \\ T_{0} & 1700(\mathrm{~K}) \\ T_{\infty} & 293(\mathrm{~K}) \\ h_{c} & 80\left(\mathrm{~W} \mathrm{~m} \mathrm{~K}^{-1}\right) \\ H_{v} & 7.34 \times 10^{6}\left(\mathrm{~J} \mathrm{~kg}^{-1}\right) \\ & \end{array}$


Table 1. Thermophysical properties of 304 stainless steel and welding conditions (cont.)

\begin{tabular}{|c|c|c|}
\hline Nomenclature & Symbol & Value (unit) \\
\hline Gas constant & $R$ & $8314.3\left(\mathrm{~J} \mathrm{~kg}^{-1} \mathrm{~mole}^{-1}\right)$ \\
\hline Stefan-Boltzmann constant & $\sigma$ & $5.67 \times 10^{-8}\left(\mathrm{~W} \mathrm{~m}^{-2} \mathrm{~K}^{-4}\right)$ \\
\hline Electrical conductivity & $\sigma_{e}$ & $7.14 \times 10^{5}\left(\Omega^{-1} \mathrm{~m}^{-1}\right)$ \\
\hline Welding voltage & $u_{w}$ & $14.7(\mathrm{~V})$ \\
\hline Welding current & $I$ & $260(\mathrm{~A})$ \\
\hline Arc heat flux distribution parameter & $\sigma_{q}$ & $2.50 \times 10^{-3}(\mathrm{~m})$ \\
\hline Arc current distribution parameter & $\sigma_{c}$ & $2.50 \times 10^{-3}(\mathrm{~m})$ \\
\hline Welding speed & $V_{a}$ & $7\left(\mathrm{~mm} \mathrm{~s}^{-1}\right)$ \\
\hline Arc thermal efficiency & $\eta$ & $80 \%$ \\
\hline Ratio of droplet thermal energy to total arc energy & $\eta_{d}$ & $48 \%$ \\
\hline Thickness of base metal & $H_{b}$ & $6.0(\mathrm{~mm})$ \\
\hline Width of base metal & $W_{b}$ & $30.0(\mathrm{~mm})$ \\
\hline Length of base metal & $L_{b}$ & $300.0(\mathrm{~mm})$ \\
\hline Initial base metal temperature & $T_{b}$ & $293(\mathrm{~K})$ \\
\hline Initial base metal sulfur concentration & $f_{b}^{\alpha}$ & 100 (ppm) \\
\hline Electrode wire diameter & $d$ & $1.60(\mathrm{~mm})$ \\
\hline Electrode wire feed speed & $V_{w}$ & $5.7(\mathrm{~cm} / \mathrm{s})$ \\
\hline Droplet diameter & $D_{d}$ & $1.50(\mathrm{~mm})$ \\
\hline Droplet generation frequency & $F_{d}$ & $65(\mathrm{~Hz})$ \\
\hline Droplet impinging velocity & $V_{d}$ & $70 \mathrm{~cm} / \mathrm{s}$ \\
\hline Initial droplet temperature & $T_{d}$ & $2500(\mathrm{~K})$ \\
\hline Initial droplet sulfur concentration & $f_{d}^{\alpha}$ & 300 (ppm) \\
\hline Maximum plasma arc pressure & $P_{\max }$ & $200(\mathrm{~Pa})$ \\
\hline Plasma arc pressure distribution parameter & $\sigma_{p}$ & $5.0 \times 10^{-3}(\mathrm{~m})$ \\
\hline
\end{tabular}




\section{FIGURE CAPTIONS}

Fig. 1. Schematic sketch of a moving GMAW system; $x-y-z$ is a fixed coordinate system and r-z is a local coordinate system moving along with the arc center.

Fig. 2. Partial view of the three-dimensional mesh system and deposition height at $t=2.564 \mathrm{~s}$.

Fig. 3. A typical sequence showing the impinging process, weld pool dynamics, and temperature distributions (side view).

Fig. 4. The corresponding velocity distributions as shown in Fig. 3.

Fig. 5. The corresponding sulfur concentration distributions as shown in Fig. 3.

Fig. 6. Cross-sectional temperature distributions at different $\mathrm{X}$ locations at $\mathrm{t}=2.938 \mathrm{~s}$ (front view).

Fig. 7. The corresponding velocity distributions as shown in Fig. 6.

Fig. 8. The corresponding sulfur concentration distributions as shown in Fig. 6.

Fig. 9. Partial view of the three-dimensional mesh system, weld bead shape, and velocity distribution at $\mathrm{t}$ $=2.938 \mathrm{~s}$.

Fig. 10. The corresponding top view of velocity, temperature, and sulfur concentration distributions as shown in Fig. 9. 